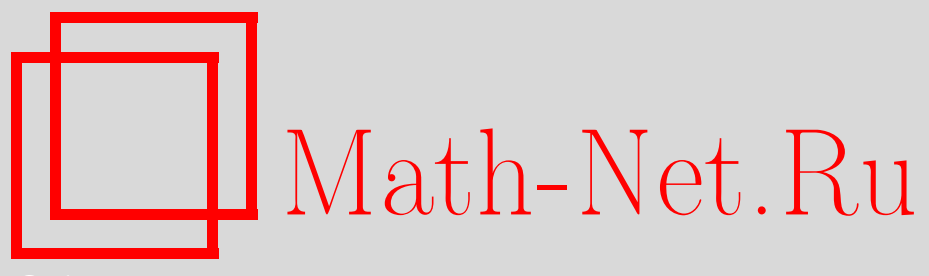

А. А. Багаев, Двухпетлевые вычисления эффективного действия матричной $\sigma$-модели в формализме фонового поля, $Т M \Phi, 2008$, том 154, номер 2, 354-362

DOI: https://doi.org/10.4213/tmf6174

Использование Общероссийского математического портала Math-Net.Ru подразумевает, что вы прочитали и согласны с пользовательским соглашением http://www.mathnet.ru/rus/agreement

Параметры загрузки:

IP : 52.90 .164 .192

26 апреля 2023 г., 12:56:31

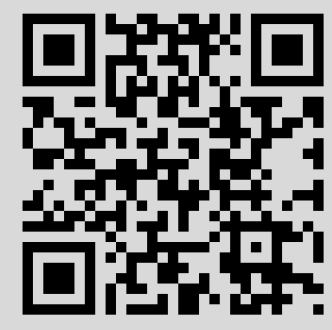




\section{ДВУХПЕТЛЕВЫЕ ВЫЧИСЛЕНИЯ ЭФФЕКТИВНОГО ДЕЙСТВИЯ МАТРИЧНОЙ $\sigma$-МОДЕЛИ В ФОРМАЛИЗМЕ ФОНОВОГО ПОЛЯ}

В формализме фонового поля рассматривается матричная $\sigma$-модель. Прямым вычислением бесконечной части эффективного действия показано равенство "бегущих констант связи" при регуляризации с импульсом обрезания и размерной регуляризации в двухпетлевом приближении. Проверено совпадение $\beta$-функции с имеющимися результатами.

Ключевые слова: фоновое поле, главное киральное поле, перенормировка, бегущая константа связи.

\section{1. ВВЕДЕНИЕ}

Двумерная матричная $\sigma$-модель (главное киральное поле) задается действием

$$
S=\frac{1}{2 e_{0}^{2}} \int_{\mathbb{R}^{2}} d^{2} x \operatorname{tr}\left(\partial_{\mu} g^{-1} \partial_{\mu} g\right),
$$

где $e_{0}$ - безразмерная константа связи, матрица $g$ - элемент компактной группы Ли $G, \operatorname{tr}$ - матричный след.

Модель (1.1) описывает двумерный голдстоуновский бозон [1], [2] и ряд явлений в теории конденсированного состояния.

Эффективное действие нашей модели в формализме фонового поля [3] имеет вид

$$
W=\frac{1}{e_{0}^{2}} W_{-1}+W_{0}+e_{0}^{2} W_{1}+\cdots+e_{0}^{2 n} W_{n}+\cdots,
$$

где $W_{n}$ - вклады $(n+1)$-петлевых вакуумных диаграмм. Все расходящиеся слагаемые (1.2) комбинируются в множитель (“бегущую константу связи") при $W_{-1}$, имеющий вид ряда по степеням $e_{0}^{2}$ с расходящимися коэффициентами [3], [4]. Из перенормируемости и асимптотической свободы [5] следует, что можно выбрать константу $e_{0}$ стремящейся к нулю при снятии регуляризации таким образом, что ряд (1.2) окажется конечным.

${ }^{*}$ Санкт-Петербургский государственный университет, Санкт-Петербург, Россия. E-mail: bagaew@mail.ru 
"Бегущая константа связи" имеет вид

$$
\frac{1}{e_{\mathrm{MC}}^{2}(\mu)}=\frac{1}{e^{2}(\Lambda)}+c_{0} \ln \frac{\Lambda}{\mu}+c_{1} \ln \frac{\Lambda}{\mu} e^{2}(\Lambda)+\cdots
$$

в регуляризации с импульсом обрезания $\Lambda$ ( $\mu$ - точка нормировки) и

$$
\frac{1}{e_{\mathrm{DR}}^{2}(\mu)}=\frac{1}{e^{2}(\varepsilon)}+\frac{b_{0}}{\varepsilon} \mu^{-\varepsilon}+\frac{b_{1}}{\varepsilon} e^{2}(\varepsilon) \mu^{-2 \varepsilon}+\cdots
$$

в размерной регуляризации, где $\varepsilon$ - дефект размерности ( $\mu$ - параметр с размерностью импульса). В старших петлях $(n \geqslant 3)$ еще имеются слагаемые, пропорциональные $(\ln \Lambda / \mu)^{n-1}$ или $\varepsilon^{1-n}$, соответственно.

Имеет место равенство $\lim _{\Lambda \rightarrow+\infty} e_{\mathrm{MC}}(\mu)=\lim _{\varepsilon \downarrow 0} e_{\mathrm{DR}}(\mu)$, если $b_{0}=c_{0}$ и

$$
c_{1}=2 b_{1}
$$

(см. работу [3]). Следовательно, $\beta$-функции, определяемые как

$$
\beta\left(e^{2}\right) \equiv \frac{d e^{2}(\mu)}{d \ln \mu}=\beta_{1} e_{0}^{4}+\beta_{2} e_{0}^{6}+\cdots,
$$

также равны, и $\beta_{1}=c_{0}, \beta_{2}=c_{1}=2 b_{1}$.

Основная цель настоящей работы - вычисление бесконечной части ряда (1.2) с точностью до двухпетлевого приближения в регуляризации с импульсом обрезания и в размерной регуляризации и проверка равенства (1.3) для $\sigma$-модели.

Фаддеев и Болохов проверяли условие (1.3) для полей Янга-Миллса, но, во-первых, вычисления оказались весьма сложными, а во-вторых, регуляризация с импульсом обрезания нарушает калибровочную инвариантность [6], [7], поэтому появляются квадратичные расходимости, не устраняемые вычитанием или перенормировкой действия. Аналогичное наблюдается и в нашем случае (см. раздел 5), но двухпетлевые вычисления с двумерной моделью (1.1) проще, чем в четырехмерной теории калибровочных полей. Структура расходимостей при этом похожа на янг-миллсовскую, а квадратичные расходимости, как будет показано, просто устраняются.

Модель (1.1) является частным случаем общей двумерной нелинейной модели (см. ниже формулу (6.2)), в которой поле принимает значения в дифференцируемом многообразии $\mathcal{M}[8]$. Для модели (6.2) известна $\beta$-функция в двухпетлевом приближении, выраженная через геометрические инварианты $\mathcal{M}$ [8], [9], однако она сложна для интерпретации. Выражение упрощается для симметрического $\mathcal{M}$ (например, $S U(N))$ [8]; будет показано, что оно совпадает с результатом нашего анализа модели (6.2) с $G=S U(N)$ в пределе большого $N$.

Подробно исследован другой частный случай модели (6.2) - $O(N)$-симметричная $\sigma$-модель (6.1), или модель $n$-поля [1], [2]; изучалось $1 / N$-разложение [5], [10]. В пределе большого $N$ теория $n$-поля описывает свободные безмассовые частицы [5]. Известна $\beta$-функция с точностью до четырехпетлевого приближения [11]. В разделе 6 показано совпадение $\beta$-функций для моделей (1.1) и (6.1), когда $G=S U(2) \cong O(4)$. 
Кроме того, $S U(N) \sigma$-модель эквивалентна интегрируемой фермионной модели [2], [12] и модели со спиновыми цепочками [13] в магнитном поле, исследованным с помощью анзаца Бете; для инвариантного заряда определен аналог $\beta$-функции (6.4), которая оказывается эквивалентной функции (1.4) для $S U(2) \sigma$-модели.

В данной работе используется формализм фонового поля [5], [4] с неклассическим внешним полем [14], что упрощает перенормировку константы связи [3]. При этом вычисления проводятся в более удобном координатном представлении, а не в традиционном импульсном (как, например, в работе [11]). Полученные коэффициенты $\beta$-функции выражены в терминах нормировки оператора Казимира группы $G$ (в рассматриваемом конкретном представлении) подобно теории Янга-Миллса [3], [4], что не встречается в современных исследованиях старших порядков для модели (1.1). Также несколько уточнены формулы [4] для двухпетлевых вычислений (см. приложение) в четырехмерном случае ${ }^{1)}$ в двух схемах регуляризации.

\section{2. ФОРМАЛИЗМ ФОНОВОГО ПОЛЯ ДЛЯ МАТРИЧНОЙ $\sigma$-МОДЕЛИ}

Представим поле $g$ в модели (1.1) в виде $g(x)=h(x) g_{\mathrm{cl}}(x)$, где $h$ - "квантовое" поле, а $g_{\mathrm{cl}}$ - "классическое" фоновое поле. Интегрирование по $h[5]$ дает формулу (1.2), $W_{0}$ - детерминант (3.1), $W_{n \geqslant 1}$ представляют собой суммы вкладов 1-непри-

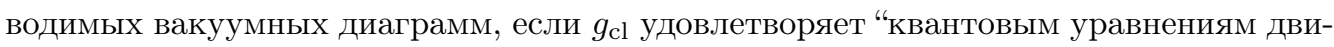
жения" [14]:

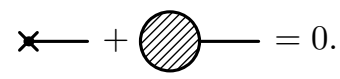

Первый член - вариационная производная (1.1), т.е. $\partial_{\mu}\left(R_{\mu}\right)_{\mathrm{cl}} \equiv \partial_{\mu}\left(\partial_{\mu} g_{\mathrm{cl}} g_{\mathrm{cl}}^{-1}\right)$, второе слагаемое - сумма 1-неприводимых графов с одной внешней линией.

Параметризуем "квантовое" поле $h(x)=e^{e_{0} \phi(x)}$ с помощью поля $\phi$, принадлежащего фундаментальному представлению алгебры $\mathfrak{g}, \phi=\phi^{A} t^{A}$, где генераторы $t^{A}$ удовлетворяют условиям

$$
\operatorname{Sp}\left(t^{A} t^{B}\right)=-\frac{1}{2} \delta^{A B}, \quad\left[t^{A}, t^{B}\right]=f^{A B C} t^{C}
$$

Здесь $f^{A B C}$ - структурные константы $\mathfrak{g}$ в фундаментальном представлении, $[\cdot, \cdot]$ коммутатор, а Sp обозначает форму Киллинга на $\mathfrak{g}$, т.е. $\operatorname{tr}(\cdot \times \cdot)$.

В этом формализме формула (1.1) переходит (детали см. в приложении) в выражение

$$
S=\frac{1}{e_{0}^{2}} W_{-1}+\sum_{n=2}^{\infty} e_{0}^{n-2} S_{n},
$$

где

$$
W_{-1}=-\frac{1}{2} \int_{\mathbb{R}^{2}} d^{2} x \operatorname{Sp}\left(R^{2}(x)\right)
$$

\footnotetext{
1) Отметим, что для случая двух измерений аппарат, аналогичный предложенному в работе [4], систематически не разработан.
} 
- классическое действие (обозначение "cl" опущено);

$$
S_{n}=-\frac{1}{n !} \int_{\mathbb{R}^{2}} d^{2} x\left[\operatorname{Sp}\left(B_{\mu}\left(\phi^{n}\right) R_{\mu}\right)-\frac{1}{2} \sum_{m=1}^{n-1}\left(\begin{array}{c}
n \\
m
\end{array}\right) \operatorname{Sp}\left(B_{\mu}\left(\phi^{n-m}\right) B_{\mu}\left(\phi^{m}\right)\right)\right]
$$

- функционалы $n$-го порядка по степеням поля $\phi\left(B_{\mu}\left(\phi^{n}\right)\right.$ - кратный коммутатор, см. формулу (П.1) в приложении); $S_{1}$ не учитывается в силу уравнений (2.1).

\section{3. ДИАГРАММНАЯ ТЕХНИКА}

Выделим из $A=e^{-S}$ слагаемые $A_{0}$ и $A_{1}$, пропорциональные $\left(e_{0}\right)^{0}$ и $\left(e_{0}\right)^{2}$ соответственно. Первое из них имеет вид

$$
A_{0}=-S_{2}=\frac{1}{2} \int_{\mathbb{R}^{2}} d^{2} x \phi^{A} K^{A B} \phi^{B},
$$

где оператор

$$
(K)^{A B}=\frac{1}{2}\left(\partial^{2} \delta^{A B}-f^{A K B} R_{\mu}^{K} \partial_{\mu}\right)
$$

задает квадратичную форму по $\phi$ в действии.

Применяя теорему Вика к $A_{1}=e_{0}^{2}\left[-S_{4}+\left(S_{3}\right)^{2} / 2\right]$, получаем $W_{1}$ - сумму вкладов диаграмм "восьмерка" и "рыбка" или пяти типов диаграмм; штрихами на линиях обозначены производные $G(x, y)$ (см. рисунок в разделе 6$)$ :

$$
\begin{aligned}
\left(W_{1}\right)_{1}=- & \frac{1}{48} f^{A B C} f^{B D E} f^{D M N} \int_{\mathbb{R}^{2}} d^{2} x R_{\mu}^{A}(x)\left[\left.\partial_{\mu} G^{M E}(x, y)\right|_{y=x} G^{N C}(x, x)+\right. \\
& +N \leftrightarrow E+C \leftrightarrow E] ; \\
\left(W_{1}\right)_{2}=- & \frac{1}{48} f^{A B C} f^{B D E} \int_{\mathbb{R}^{2}} d^{2} x\left[\left.\left.\partial_{\mu} G^{D E}(x, y)\right|_{y=x} \partial_{\mu} G^{A C}(x, y)\right|_{y=x}+C \leftrightarrow E\right] ; \\
\left(W_{1}\right)_{3}=- & \frac{1}{48} f^{A B C} f^{B D E} \int_{\mathbb{R}^{2}} d^{2} x\left[\left.G^{E C}(x, x) \partial_{\mu} G^{D A}(x, y) \overleftarrow{\partial}\right|_{y=x}\right] ; \\
\left(W_{1}\right)_{4}= & \frac{1}{288} f^{A K C} f^{K M E} f^{B L D} f^{L N F} \int_{\mathbb{R}^{2}} \int_{\mathbb{R}^{2}} d^{2} x d^{2} y \times \\
& \times\left[\partial_{\mu} G^{M N}(x, y) \overleftarrow{\partial}{ }_{\nu} G^{E F}(x, y) G^{C D}(x, y)+D \leftrightarrow F+F \leftrightarrow N\right] R_{\mu}^{A}(x) R_{\nu}^{B}(y) \\
\left(W_{1}\right)_{5}= & \frac{1}{288} f^{A K C} f^{K M E} f^{B L D} f^{L N F} \int_{\mathbb{R}^{2}} \int_{\mathbb{R}^{2}} d^{2} x d^{2} y\left[\partial_{\mu} G^{M D}(x, y) G^{C N}(x, y) \overleftarrow{\partial}{ }_{\nu} \times\right. \\
& \left.\times G^{E F}(x, y)+2 \cdot D \leftrightarrow F\right] R_{\mu}^{A}(x) R_{\nu}^{B}(y) .
\end{aligned}
$$

\section{4. ВЫЧИСЛЕНИЯ В ОДНОПЕТЛЕВОМ ПРИБЛИЖЕНИИ}

Известно, что $W_{0}=(1 / 2) \ln (\operatorname{det} K)$. Вычислим его методом теплового ядра (или собственного времени Фока) [15]-[17] с помощью формулы (следующей из (П.2), см. приложение)

$$
\ln \operatorname{det} K=\mathrm{const}-\int_{0}^{+\infty} \frac{d s}{s}\left(\int_{\mathbb{R}^{2}} d^{2} x \theta^{A B}(x, x, s)\right),
$$


где $\theta(x, y, s)$ - фундаментальное решение $(3.1)$, известное в виде разложения в окрестности точки $s=0[15]$ :

$$
\theta^{A B}(x, y, s)=\frac{1}{4 \pi s} e^{-(x-y)^{2} /(4 s)}\left[a_{0}^{A B}(x, y)+a_{1}^{A B}(x, y) s+\cdots\right], \quad a_{0}^{A B}(x, x)=\delta^{A B} .
$$

Гладкие коэффициенты $a_{n}^{A B}(x, y)$ удовлетворяют уравнениям (П.3) (см. приложение).

Регуляризуем (4.1), заменив расходящийся интеграл на логарифм; из явного вида оператора (3.1), формулы (П.3б) и производных по $x_{\mu}$ уравнения (П.3а) (см. приложение) получаем, что бесконечная часть $W_{0}$ имеет вид

$$
\widetilde{W}_{0}=-\frac{1}{2 \pi} \int_{\mathbb{R}^{2}} d^{2} x a_{1}^{A A}(x, x) \cdot \ln \frac{\Lambda}{\mu}=-\frac{C(G)}{4 \pi} W_{-1} \ln \frac{\Lambda}{\mu},
$$

где $C(G)$ - собственное значение оператора Казимира алгебры g. Следовательно, $c_{0}=b_{0}=-C(G) /(4 \pi)$, что совпадает с результатами, полученными в монографии [5].

\section{5. ВЫЧИСЛЕНИЯ В ДВУХПЕТЛЕВОМ ПРИБЛИЖЕНИИ}

Функция Грина $G^{A B}(x, y)$ оператора (3.1) связана с функцией $\theta(4.2)$ соотношением

$$
G^{A B}(x, y)=\int_{0}^{+\infty} d s \theta^{A B}(x, y, s)=a_{0}^{A B}(x, y) p_{0}(x-y)+a_{1}^{A B}(x, y) p_{1}(x-y)+\cdots
$$

где $p_{0}(x)$ имеет сингулярность высшего порядка при $x=0,\left[p_{1}\right]=\left[p_{0}\right]+2$.

Есть три вида расходимостей (3.2):

1) $p_{0}^{2}, \quad \partial_{\mu} p_{0} \partial_{\mu} p_{1}, \quad p_{0}, \partial^{2} p_{1} p_{0}, \quad \int d y \partial_{\mu} p_{0} \partial_{\nu} p_{0} p_{0}, \quad \int d y \partial_{\mu} \partial_{\nu} p_{0} p_{0}^{2}, \quad \int d y \partial_{\mu} p_{0} \partial_{\nu} p_{0}$, $\int d y \partial_{\mu} \partial_{\nu} p_{0} p_{0}$ имеют размерность $\left[p_{0}\right]$;

2) $\partial_{\mu} p_{0} p_{0}, \partial_{\mu} p_{0}$ имеют размерность $\left[p_{0}\right]-1$;

3) $\partial_{\mu} p_{0} \partial_{\mu} p_{0}, \partial^{2} p_{0} p_{0}, \partial^{2} p_{0}$ имеют размерность $\left[p_{0}\right]-2$.

В случае двумерного пространства первый из множителей в формуле (5.1),

$$
p_{0}(x)=-\frac{1}{4 \pi} \ln \mu^{2} x^{2}, \quad p_{1}(x)=\frac{1}{16 \pi} x^{2}\left[\ln \mu^{2} x^{2}-1\right]
$$

(ср. с работой [4]), имеет особенность в точке $x=0$. В окончательных выражениях осуществим замену $x^{2} \mapsto \epsilon^{2} \sim 1 / \Lambda^{2}$; в этом состоит регуляризация с импульсом обрезания.

Все расходимости являются либо квадратичными, либо линейными, либо логарифмическими по параметру $\Lambda:\left\langle\widetilde{W}_{1}\right\rangle_{\mathrm{MC}}=\Gamma_{2}+\Gamma_{1}+\Gamma_{0}$.

Квадратичная расходимость

$$
\Gamma_{2}=\left.\frac{1}{12} C(G) \int_{\mathbb{R}^{2}} d^{2} x F^{A A}(x, x) \cdot \partial^{2} p_{0}(y)\right|_{y=0}
$$


присутствует, как и в теории Янга-Миллса. Функционал, пропорциональный квадратично-расходящемуся множителю, является "калибровочно-инвариантным" объектом, если предположить, что поля $R_{\mu}(x)$ играют роль связности, как и калибровочные поля. Это предположение резонно благодаря уравнению параллельного переноса (П.3а) и структуре оператора (П.4) (см. приложение). То же самое наблюдается и при анализе полей Янга-Миллса: квадратичные расходимости возникают вследствие того, что регуляризация с импульсом обрезания не является калибровочно-инвариантной [6], [7]. Однако заметим, что

$$
\begin{array}{r}
\left.\frac{1}{12} f^{A B M} f^{M D C} \int_{\mathbb{R}^{2}} d^{2} x G^{B D}(x, x) K^{A L} G^{L C}(x, y)\right|_{y=x}= \\
=\text { const }+\left.\frac{1}{12} C(G) \int_{\mathbb{R}^{2}} d^{2} x F^{A A}(x, x) \cdot \partial^{2} p_{0}(y)\right|_{y=0}
\end{array}
$$

значит,

$$
\widetilde{W}_{1}^{\prime}=\widetilde{W}_{1}-\left.\frac{1}{12} f^{A B M} f^{M D C} \int_{\mathbb{R}^{2}} d^{2} x G^{B D}(x, x) K^{A L} G^{L C}(x, y)\right|_{y=x}
$$

не содержит квадратичных расходимостей, при том что остальные расходимости $\widetilde{W_{1}^{\prime}}$ и $\widetilde{W}_{1}$ одинаковы. В случае Янга-Миллса аналогичный прием не применим.

Выражение $\Gamma_{1}$, пропорциональное $\left.\partial_{\mu} p_{0}(y)\right|_{y=0}$, равно нулю в силу четности выражения (5.2).

Логарифмический вклад $\Gamma_{0}=\Gamma_{0}^{2}+\Gamma_{0}^{1}-$ сумма квадрата и первой степени логарифма; $\Gamma_{0}^{2}=0$, что следует из (П.5а) для случая МС, а $\Gamma_{0}^{1}=\left\langle\widetilde{W}_{1}^{\prime}\right\rangle_{\mathrm{MC}}$ с помощью (П.5б) и (П.5в) (см. приложение) запишется как

$$
\left\langle\widetilde{W}_{1}^{\prime}\right\rangle_{\mathrm{MC}}=-\frac{1}{3} C^{2}(G)\left(\frac{p_{0}(0)}{16 \pi}+\left.\frac{1}{2} \partial_{\mu} p_{0}(x) \partial_{\mu} p_{1}(x)\right|_{x=0}\right) W_{-1}=-\frac{C^{2}(G)}{32 \pi^{2}} W_{-1} \ln \frac{\Lambda}{\mu},
$$

следовательно, $c_{1}=-C^{2}(G) /\left(32 \pi^{2}\right)$.

Для размерной регуляризации перейдем формально в пространство $2-\varepsilon$ измерений. Множители в соотношении (5.1) имеют вид

$$
\begin{aligned}
& p_{0}(x)=\frac{1}{4 \pi}\left\{\frac{2}{\varepsilon} \mu^{-\varepsilon}+\pi^{\varepsilon / 2} \Gamma\left(-\frac{\varepsilon}{2}\right)|x|^{\varepsilon}\right\}, \\
& p_{1}(x)=\frac{1}{16 \pi}\left\{-\frac{2 x^{2}}{\varepsilon} \mu^{-\varepsilon}+\pi^{\varepsilon / 2} \Gamma\left(-1-\frac{\varepsilon}{2}\right)|x|^{2+\varepsilon}\right\} .
\end{aligned}
$$

Расходимости - это полюсы по $\varepsilon ;$ в двух петлях имеются полюсы только первого и второго порядков, причем вклад, пропорциональный $1 / \varepsilon^{2}$, равен нулю, как и $\Gamma_{0}^{2}$ в регуляризации с импульсом обрезания.

Для $1 / \varepsilon$ (с учетом (П.5), см. приложение) получается

$$
\left\langle W_{1}\right\rangle_{\mathrm{DR}}=-\frac{1}{3} C^{2}(G)\left(\frac{\mu^{-\varepsilon}}{16 \pi} p_{0}(0)+\frac{\mu^{-2 \varepsilon}}{64 \pi^{2}}\right) W_{-1}=-\frac{C^{2}(G)}{64 \pi^{2}} W_{-1} \frac{\mu^{-2 \varepsilon}}{\varepsilon},
$$

откуда $b_{1}=-C^{2}(G) /\left(64 \pi^{2}\right)$. 


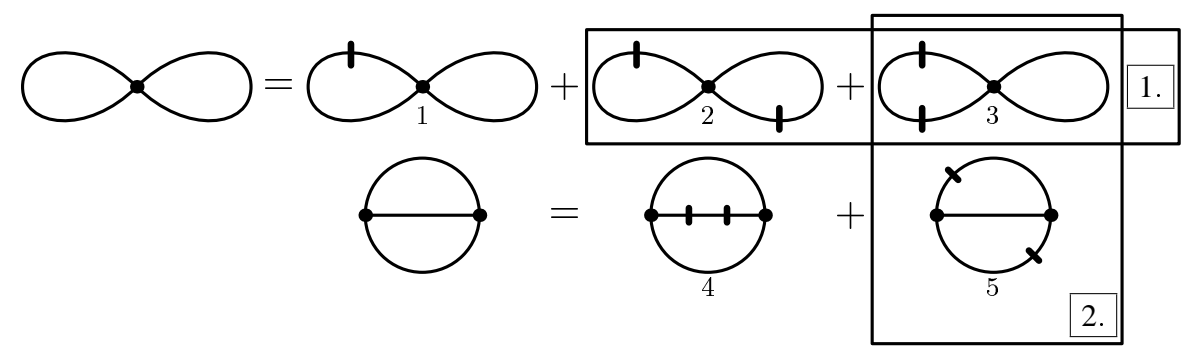

Различие между двумя схемами регуляризации на языке диаграмм: 1. регуляризация с импульсом обрезания; 2 . - размерная регуляризация; 1,2 , 3, 4, 5 - номера слагаемых (3.2).

\section{6. ВЫВОДЫ}

Сравнивая формулы (5.3) и (5.4), видим, что для $c_{1}$ и $b_{1}$ выполняется равенство (1.3).

Схема на рисунке иллюстрирует состав слагаемых (3.2), дающих логарифмический вклад в эффективное действие в различных схемах регуляризации.

Для $O(N)$-симметричной $\sigma$-модели с действием

$$
S=\frac{1}{2 e_{0}^{2}} \int_{\mathbb{R}^{2}} d^{2} x\left(\partial_{\mu} \mathbf{n}, \partial_{\mu} \mathbf{n}\right), \quad(\mathbf{n}, \mathbf{n})=1, \quad \mathbf{n} \in \mathbb{R}^{N},
$$

где $(\cdot, \cdot)$ - скалярное произведение в пространстве $\mathbb{R}^{N}$, известна $\beta$-функция [11]

$$
\beta(T)=\varepsilon T-(N-2) T^{2}-(N-2) T^{3}+O\left(T^{4}\right),
$$

где $T$ - температура, аналог константы связи. Эквивалентность $O(4)-$ и $S U(2)$ $\sigma$-моделей показана в приложении. Сравним $\beta$-функции.

Приравнивая $4 \pi \beta(T)^{2)}$ при $N=4$ и выражение (1.4) с коэффициентами (4.3) и (5.3) при $C(G)=2$, находим, что $T=e_{0}^{2} /(4 \pi)$. Следовательно, в данном частном случае результаты настоящей работы и работы [11] совпадают.

Для общей нелинейной двумерной модели

$$
S[X]=\frac{1}{2} \int_{\mathbb{R}^{2}} d^{2} x G_{a b}(X) \partial_{\mu} X^{a} \partial_{\mu} X^{b},
$$

когда $X(x) \in \mathcal{M}$, уравнения Гелл-Манна-Лоу можно найти в монографии [9]. В частном случае симметрического многообразия, т.е. когда $\mathcal{M}=G / H$, имеем

$$
\beta_{a b}\left(\frac{G}{T}\right)=\left(-\varepsilon+\frac{1}{2} T+\frac{(\operatorname{dim} \mathcal{M})^{2}+4 \operatorname{dim} A(\operatorname{dim} H-\operatorname{dim} \mathcal{M})}{8 \operatorname{dim} \mathcal{M}(\operatorname{dim} H-\operatorname{dim} A)} T^{2}\right) \frac{G_{a b}}{T}
$$

(см. работу [8]), где в случае $S U(N) \sigma$-модели $G=S U(N) \times S U(N), H=S U(N)$, $\mathcal{M}=S U(N), \operatorname{a} \operatorname{dim} A$ есть размерность подалгебры Картана $H$, т.е. $N-2$. Если принять

$$
T=\frac{C(G)}{2 \pi} e_{0}^{2}=\frac{N}{2 \pi} e_{0}^{2},
$$

\footnotetext{
2) Дополнительные множители - от $\beta_{1}=-(N-2) /(2 \pi)$ для $O(N) \sigma$-модели [5] и от (П.6).
} 
то выражение (6.3) будет давать (1.4) в режиме больших $N$.

Формула для инвариантного заряда $Z$ спиновой цепочки во внешнем магнитном поле $h$ (см. формулу (5.9) в работе [13])

$$
\frac{d Z}{d L}=\beta(Z)=-\frac{Z^{2}}{\pi}-\frac{Z^{3}}{2 \pi^{2}}+\cdots, \quad L=\ln \frac{h}{m},
$$

где $m$ - параметр, имеющий смысл массы, получена в результате анализа $S U(2)$ $\sigma$-модели с помощью анзаца Бете. Если принять $Z=e_{0}^{2} / 2$, то имеет место равенство $2 \beta(Z)=\beta_{1} e_{0}^{4}+\beta_{2} e_{0}^{6}+\cdots$, в котором $C(G)=2$. Множитель 2 может быть устранен переопределением действия.

\section{ПРИЛОЖЕНИЕ}

Используя $L_{\mu}=h^{-1} \partial_{\mu} h$, перепишем подынтегральное выражение в формуле (1.1) в виде

$$
\begin{aligned}
\operatorname{tr}\left(\partial_{\mu} g^{-1} \partial_{\mu} g\right)=\operatorname{Sp}( & \left.\left(R_{\mathrm{cl}}+L\right)^{2}\right)=-\operatorname{Sp}\left(\left(R_{\mu}\right)_{\mathrm{cl}}\left(R_{\mu}\right)_{\mathrm{cl}}\right)- \\
& -2 \operatorname{Sp}\left(\left(R_{\mu}\right)_{\mathrm{cl}} \cdot h^{-1} \partial_{\mu} h\right)-\operatorname{Sp}\left(h^{-1} \partial_{\mu} h \cdot h^{-1} \partial_{\mu} h\right) .
\end{aligned}
$$

Комбинация $h^{-1} \partial_{\mu} h$ имеет вид

$$
h^{-1} \partial_{\mu} h=\sum_{n=1}^{\infty} \frac{e_{0}^{n}}{n !}\left[\ldots[\partial_{\mu} \underbrace{\phi, \phi], \ldots, \phi}_{n \text { раз }}] ;\right.
$$

мы использовали известное правило дифференцирования экспоненты [18].

Для вычислений с помощью метода собственного времени полезна следующая формула:

$$
\int_{0}^{+\infty} d x \frac{e^{-x}-e^{-A x}}{x}=\ln A, \quad A>0 .
$$

Коэффициенты (4.2) и (5.1) удовлетворяют цепочке рекуррентных уравнений

$$
\begin{gathered}
-\left(x_{\mu}-y_{\mu}\right) M_{\mu}^{A C} a_{0}^{C B}=0, \\
-\left(x_{\mu}-y_{\mu}\right) M_{\mu}^{A C} a_{N+1}^{C B}+K^{A C} a_{N}^{C B}=(N+1) a_{N+1}^{A B},
\end{gathered}
$$

где введен новый оператор

$$
M_{\mu}^{A B}=\partial_{\mu} \delta^{A B}-\frac{1}{2} f^{A K B} R_{\mu}^{K} .
$$

Для двухпетлевых вычислений использованы формулы

$$
\begin{gathered}
\int_{\mathbb{R}^{2}} d y \partial_{\mu} \partial_{\nu} p_{0}(y) p_{0}^{2}(y)= \begin{cases}-\frac{1}{2} p_{0}^{2}(0) \delta_{\mu \nu} & \text { в случае MC, } \\
-\frac{1}{8 \pi^{2}} \frac{\mu^{-2 \varepsilon}}{\varepsilon^{2}} \delta_{\mu \nu} & \text { в случае DR; }\end{cases} \\
\int_{\mathbb{R}^{2}} d y \partial_{\mu} p_{0}(y) \partial_{\nu} p_{0}(y) p_{0}(y)= \begin{cases}\frac{1}{4} p_{0}^{2}(0) \delta_{\mu \nu} & \text { в случае MC, } \\
\frac{\mu^{-2 \varepsilon}}{16 \pi^{2}}\left[\frac{1}{\varepsilon^{2}}-\frac{1}{2 \varepsilon}\right] \delta_{\mu \nu} & \text { в случае DR; }\end{cases} \\
\partial^{2} p_{1}=-p_{0}+\frac{\mu^{-\varepsilon}}{4 \pi}
\end{gathered}
$$


(ср. с работой [4]), а также формула

$$
\left.\partial_{\mu} p_{0}(x) \partial_{\mu} p_{1}(x)\right|_{x=0}= \begin{cases}\frac{1}{4 \pi} p_{0}(0) & \text { в случае MC, } \\ 0 & \text { в случае DR, }\end{cases}
$$

которая выполняется и в четырехмерии для $p_{1}$ и $p_{2}$ вместо $p_{0}$ и $p_{1}$ соответственно (с квадратом коэффициента); однако подобная комбинация не рассматривалась в работе [4].

Очевидно, что $S U(2) \ni g=a I+2 i\langle\vec{b}, \vec{\sigma}\rangle$, где $\mathbf{n}=(a ; \vec{b})$ - единичный четырехмерный вектор, $\langle\cdot, \cdot\rangle$ - скалярное произведение в $\mathbb{R}^{3}, \vec{\sigma}$ - вектор, составленный из матриц Паули, а $I$ - единичная матрица в пространстве $\mathbb{C}^{2}$.

Подынтегральное выражение в формуле (1.1)

$$
\operatorname{tr}\left(\partial_{\mu} g^{-1} \partial_{\mu} g\right)=\operatorname{tr}\left(\left[\partial_{\mu} a \partial_{\mu} a+\left\langle\partial_{\mu} \vec{b}, \partial_{\mu} \vec{b}\right\rangle\right] I\right)=2\left(\partial_{\mu} \mathbf{n}, \partial_{\mu} \mathbf{n}\right)
$$

с точностью до константы совпадает с подынтегральным выражением (6.1).

Благодарности. Автор благодарит Т. А. Болохова и Л.Д. Фаддеева за обсуждение изучаемой проблемы и ценные замечания.

\section{Список литературы}

[1] A. M. Polyakov, Phys. Lett. B, 72 (1977), 224.

[2] A. Polyakov, P. B. Wiegmann, Phys. Lett. B, 131 (1983), 121.

[3] Л. Д. Фаддеев, ТМФ, 148:1 (2006), 133.

[4] I. Jack, H. Osborn, Nucl. Phys. B, 207 (1982), 474.

[5] А. М. Поляков, Калибровочнъе поля и струны, Удмуртский ун-т, Ижевск, 1999.

[6] A. A. Slavnov, Phys. Lett. B, 518 (2001), 195.

[7] А. А. Славнов, ТМФ, 130:1 (2002), 3.

[8] D. Friedan, Ann. Phys., 163 (1985), 318.

[9] А. М. Цвелик, Квантовая теория поля в физике конденсированного состояния, Физматлит, М., 2002.

[10] E. Brézen, J. Zinn-Justin, Phys. Rev. B, 14 (1976), 3110.

[11] S. Hikami, E. Brezen, J. Phys. A, 11 (1978), 1141.

[12] P. B. Wiegmann, Phys. Lett. B, 141 (1984), 217.

[13] L. D. Faddeev, N. Yu. Reshetikhin, Ann. Phys., 167 (1986), 227.

[14] L. Faddeev, Bull. Braz. Math. Soc. (N.S.), 33:2, 201.

[15] Б. С. Девитт, Динамическая теория групп и полей, Наука, М., 1987.

[16] V.A. Fock, Phys. Z. Sowjetunion, 12 (1937), 404.

[17] J. Schwinger, Phys. Rev., 82 (1951), 664.

[18] М. М. Постников, Группы и алгебры Ли, Лекции по геометрии. Семестр 5, Наука, М., 1982.

Поступила в редакцию 28.12.2006, после доработки 16.03.2007 